\title{
Switching to Degludec is Associated with Reduced Hypoglycaemia, Irrespective of Definition Used or Patient Characteristics: Secondary Analysis of the ReFLeCT Prospective, Observational Study
}

\author{
Harold W. de Valk (D) - Michael Feher - Troels Krarup Hansen • \\ Johan Jendle · Mette Marie Koefoed - Ehsan Parvaresh Rizi • \\ Esther Zimmermann · Gian Paolo Fadini
}

Received: April 15, 2020 / Published online: July 14, 2020

(C) The Author(s) 2020

\begin{abstract}
Introduction: Hypoglycaemia is a common side effect of insulin therapy; low or high glycated haemoglobin $\left(\mathrm{HbA}_{1 \mathrm{c}}\right)$ levels, history of hypoglycaemia or long diabetes duration are known modifiers of hypoglycaemia risk. In randomised clinical trials, lower rates of hypoglycaemia have been observed with the newgeneration insulin analogue, long-acting insulin degludec, compared with other basal insulins.
\end{abstract}

Digital Features To view digital features for your article go to https://doi.org/10.6084/m9.figshare. 12563717.

Electronic Supplementary Material The online version of this article (https://doi.org/10.1007/s13300020-00875-1) contains supplementary material, which is available to authorized users.

H. W. de Valk $(\bowtie)$

Department of Internal Medicine, University

Medical Center Utrecht, Utrecht, The Netherlands

e-mail: H.W.deValk@umcutrecht.nl

M. Feher

Beta Cell Diabetes Centre, Chelsea and Westminster

Hospital, London, UK

M. Feher

University of Surrey, Guildford, UK

Present Address:

M. Feher

Nuffield Department of Primary Care Health

Sciences, University of Oxford, Oxford, UK
Methods: The ReFLeCT study was a prospective observational study over 12 months. Patient-reported diary data on hypoglycaemia were collected from patients with type 1 diabetes (T1D) or type 2 diabetes (T2D) who were switching from other basal insulins to insulin degludec (degludec) at their physician's discretion in routine clinical care. Two secondary analyses were undertaken to investigate the change in number of hypoglycaemic events: a post hoc analysis using the updated American Diabetes Association (ADA) level 1, 2 and 3 hypoglycaemia definitions, and a pre-specified analysis using patient characteristics (baseline $\mathrm{HbA}_{1 \mathrm{c}}$, diabetes duration, and physician's rationale for initiating degludec).

Results: Switching to degludec was associated with significantly fewer hypoglycaemic events for all definitions in T1D, and level 1 and 2 in

\section{T. K. Hansen}

Steno Diabetes Center Aarhus, Aarhus University Hospital, Aarhus, Denmark

\section{J. Jendle}

Faculty of Medicine and Health, School of Medical Sciences, Örebro University, Örebro, Sweden

M. M. Koefoed · E. P. Rizi · E. Zimmermann Novo Nordisk A/S, Søborg, Denmark

G. P. Fadini

Department of Medicine, Division of Metabolic Diseases, University of Padova, Padova, Italy 
T2D (too few level 3 events for statistical comparison). Moreover, patient characteristics did not influence the observed reduction in hypoglycaemia in T1D and T2D.

Conclusion: These results demonstrate that switching to degludec from other basal insulins was associated with reduced rates of hypoglycaemia, irrespective of the definition used or baseline patient characteristics.

Trial Registration: NCT02392117

\section{PLAIN LANGUAGE SUMMARY}

Low blood sugar levels (hypoglycaemia) are a common, and sometimes serious, side effect of treatment with insulin in people with diabetes. In the ReFleCT study, adults with type 1 (T1D) and type 2 diabetes (T2D) were asked to complete a diary for 12 months when their doctor changed their previous long-acting insulin treatment to insulin degludec (degludec). The key outcome of the study was whether the frequency of hypoglycaemia changed when a patient's insulin treatment was switched. Here, we used the diary information from the ReFLeCT study to investigate whether the change in the rate of hypoglycaemia was related to the way hypoglycaemia was defined, or to patients' characteristics at the time their insulin was switched. These characteristics included the length of time that patients had had diabetes, their blood sugar control, and their doctor's reason for changing their medication. Our findings showed that the way hypoglycaemia was defined, and patients' characteristics, did not generally influence the frequency of hypoglycaemia for patients with T1D or T2D. However, the most severe hypoglycaemia in patients with T2D occurred too infrequently to be assessed. Patients in all groups had less hypoglycaemia overall after switching compared with their previous treatment, suggesting that degludec may be a treatment option for a broad range of patients with diabetes.
Keywords: Basal insulin; Insulin degludec; Hypoglycaemia; Type 1 diabetes; Type 2 diabetes

\section{Key Summary Points}

Hypoglycaemia is a common problem for people with diabetes receiving insulin therapy, which may limit the achievement of glycaemic targets, and is associated with morbidity and long-term complications.

In the prospective observational ReFLeCT study, patient-reported diary data were used to demonstrate that patients with type 1 and type 2 diabetes (T2D) switched to insulin degludec (degludec) from another basal insulin experienced significantly less hypoglycaemia over 12 months.

In this secondary analysis, we investigated whether this effect was consistent with the use of the updated American Diabetes Association (ADA) hypoglycaemia definitions, and when assessed by patients' baseline characteristics.

Patients experienced significantly less hypoglycaemia overall with degludec regardless of the definition used, or their baseline duration of diabetes, baseline HbA1c or physician's reason for switching to degludec. However, there were too few episodes of level 3 hypoglycaemic episodes in patients with T2D for these to be assessed.

These findings suggest that switching to degludec from other basal insulins may be associated with lower hypoglycaemia rates in a broad range of patients in routine clinical practice. 


\section{INTRODUCTION}

Hypoglycaemia is a common and potentially serious treatment side effect of insulin therapy in both type 1 (T1D) and type 2 diabetes (T2D) [1]. Randomised controlled trials (RCTs) have demonstrated a hypoglycaemia risk reduction with insulin degludec (degludec), a new-generation long-acting basal insulin analogue, compared with insulin glargine 100 units $/ \mathrm{mL}$ (U100) in both T1D and T2D [2-5]. The Results from Real-World Clinical Treatment with Tresiba ${ }^{\circledR}$ (ReFLeCT) study was a prospective, observational study that collected real-world data on hypoglycaemia and other clinical parameters of patients receiving degludec in routine clinical practice [6]. In the ReFLeCT study, switching to degludec from other basal insulins was associated with significantly reduced rates of hypoglycaemia in insulin-treated adults with T1D or T2D [6].

The International Hypoglycaemia Study Group (IHSG) developed new hypoglycaemia definitions in 2016, endorsed by the American Diabetes Association (ADA) and the European Association for the Study of Diabetes (EASD) [7]. The ADA issued updated definitions in 2019 [8]. The IHSG developed the updated definitions to standardise reporting across clinical trials, and allow for improved cross-trial comparisons [7]. These definitions were developed after data collection started in the ReFLeCT study in 2015 [6].

Clinical risk factors for hypoglycaemia include older age, exercise, renal impairment, hypoglycaemia-induced autonomic failure, and intensification of glycaemic treatment (and the degree of glycaemic control) [1]. Many RCTs exclude patients with these risk factors, recurrent/recent severe hypoglycaemia [5, 9-11] or $\mathrm{HbA}_{1 \mathrm{c}}$ levels $<7.0 \%[5,10],>9.5 \%$ or $>10 \%$ $[3-5,10]$. Consequently, there is a paucity of research in these populations, despite a notably high hypoglycaemia risk in some groups (e.g. those with $\mathrm{HbA}_{1 \mathrm{c}}$ levels $<6.5 \%$ or $>9.0 \%$ ) [1]. The ReFLeCT study applied very few inclusion and exclusion criteria. This provides an opportunity to investigate whether treatment with degludec is likely to benefit patients not typically included in RCTs, e.g. because of their baseline $\mathrm{HbA}_{1 \mathrm{c}}$ or high hypoglycaemia risk, as indicated by long diabetes duration [1] or recent hypoglycaemia.

The aim of these secondary analyses was to investigate the change in the number of hypoglycaemic events in the ReFLeCT study using updated hypoglycaemia definitions (post hoc analysis), and to reanalyse the primary endpoint across patient characteristics (pre-specified analysis).

\section{METHODS}

\section{Overview}

The detailed methods of the study have been published previously [6]. In brief, the ReFLeCT study was a prospective, observational study, comprising a 4-week baseline period on patients' current basal insulin and a 12-month follow-up period after switching to degludec 100 units/mL or 200 units/mL [6]. Insulin-treated patients, aged 18 years or more with T1D or T2D, whose physician decided to switch them to degludec treatment, were eligible for inclusion [6]. The final decision to initiate degludec occurred within routine clinical practice [6]. The study was conducted across seven countries: Denmark, Italy, the Netherlands, Spain, Sweden, Switzerland and the United Kingdom (UK) [6]. Informed consent was obtained from all patients, in accordance with the requirements of the Declaration of Helsinki, before any study-related activities [12]. Independent ethics committees and institutional review boards across the participating centres reviewed and approved this study (Supplementary Table S1).

Data on hypoglycaemic events experienced (date/time of event, if it was self-treated, symptoms experienced, blood glucose [BG] value [if recorded], and resource use) were collected prospectively using dedicated patient diaries [6]. Patient diary data were collected during four discrete periods, occurring at regular intervals during the 12-month duration of follow-up, and each lasting 4 weeks. The primary endpoint of the ReFLeCT study was the change from the baseline period in the number 
of overall hypoglycaemic events during the 12-month follow-up period. Overall hypoglycaemia was defined as any event recorded as hypoglycaemia in the diary, irrespective of symptoms, BG measurement or time of day [6].

These analyses investigated the change in the number of hypoglycaemic events after switching to degludec from other basal insulins using updated hypoglycaemia definitions (post hoc analysis) and pre-specified patient characteristics. Hypoglycaemia rates were compared between the 4-week baseline period and the 4-week data collection periods during 12 months' follow-up after switching to degludec.

\section{Updated ADA Hypoglycaemia Definitions}

Updated ADA hypoglycaemia definitions were used to investigate the change in the number of hypoglycaemic events after switching to degludec from other basal insulins; level 1, $\mathrm{BG} \geq 3.0-<3.9 \mathrm{mmol} / \mathrm{L} \quad(\geq 54-<70 \mathrm{mg} / \mathrm{dL})$; level 2 , BG $<3.0 \mathrm{mmol} / \mathrm{L}(<54 \mathrm{mg} / \mathrm{dL})$; level 3 (severe), an event with severe cognitive impairment that required external assistance [8].

\section{Pre-specified Patient Characteristics}

Secondary analyses were carried out investigating the change in the number of overall hypoglycaemic events after switching to degludec from other basal insulins, using pre-specified patient characteristics. Patients were categorised according to baseline $\mathrm{HbA}_{1 \mathrm{c}}$ level, diabetes duration quartiles, and physician's reason for initiating degludec (concern about hypoglycaemia or not hypoglycaemia-related).

\section{Statistical Analyses}

Data were analysed separately for patients with T1D and T2D, and the number of hypoglycaemic events was converted to rates per patient-year of exposure (PYE). In both analyses, rate ratios (RRs) for hypoglycaemia between the 4-week baseline and 12-month follow-up periods were analysed using negative binomial regression specifying a log-transformed followup time offset term adjusted for baseline covariates; these were also used to test for interactions between period and subgroups for overall hypoglycaemia. Baseline covariates included period (pre/post-switch to degludec), baseline $\mathrm{HbA}_{1 \mathrm{c}}$ level, gender, body mass index, duration of diabetes, age and country. Additional T2D covariates were bolus insulin (Yes/ No) and sulfonylureas or glinides (Yes/No). All statistical tests were two-sided with a significance level of $p<0.05$.

\section{RESULTS}

The primary endpoint of the ReFLeCT study demonstrated that switching to degludec from other basal insulins was associated with significantly reduced rates of overall hypoglycaemia in insulin-treated adults with T1D (RR 0.80 $\left.[0.74 ; 0.88]_{95 \% \text { confidence interval }[\mathrm{CI}]}\right)$ and T2D (RR $\left.0.46[0.38 ; 0.56]_{95 \% \mathrm{CI}}\right)$. Mean $\mathrm{HbA}_{1 \mathrm{c}}$ decreased significantly from baseline to the follow-up period for patients with T1D or T2D (Supplementary Table S2). Baseline characteristics and reasons for switching to degludec are presented in Supplementary Tables S3 and S4, respectively. Insulin glargine (U100 and U300 [300 units/mL], individuals' concentration not recorded) was the most common pre-trial basal insulin in patients with T1D and T2D $(63.8 \%$ and 59.1\%, respectively; Supplementary Table S3).

\section{Hypoglycaemia Rates Using Updated ADA Hypoglycaemia Definitions}

Overall, 481 and 516 patients with T1D and T2D, respectively, were included in the fully adjusted analysis, with diary and complete covariate data. For patients with T1D, RRs demonstrated significantly lower rates of hypoglycaemia during the 12-month follow-up, versus the 4-week baseline period, for the level 1 (RR $0.90[0.81 ; 0.99]_{95 \% \mathrm{CI}}$ ), level 2 (RR 0.80 $[0.70 ; 0.91]_{95 \% \mathrm{CI}}$ ) and level 3 (RR 0.28 [0.14; $0.56]_{95 \% \mathrm{CI}}$ ) hypoglycaemia definitions (Fig. 1).

Similarly, for patients with T2D, RRs showed significantly lower rates of hypoglycaemia 


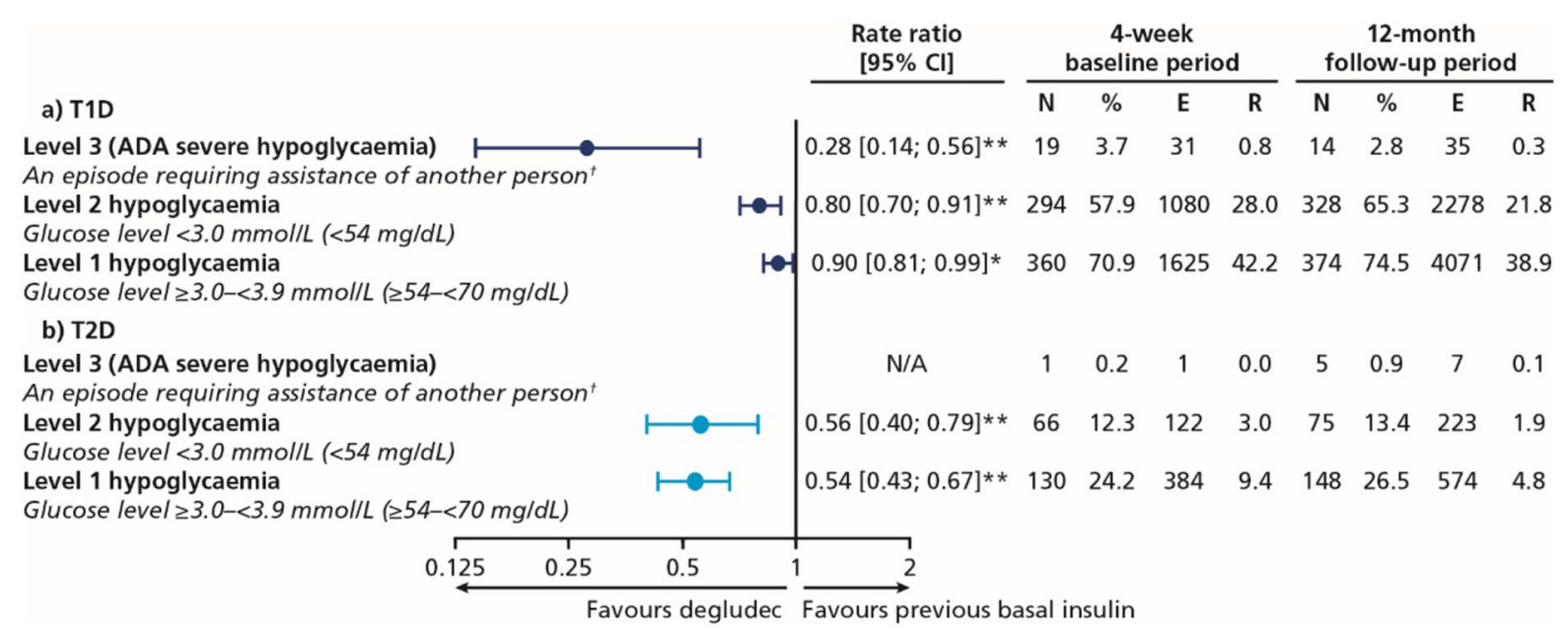

Fig. 1 Rate ratios of hypoglycaemia according to updated hypoglycaemia definitions in patients with a T1D and b T2D. ${ }^{*} p<0.05 ;{ }^{* *} p<0.001$. 'Severe hypoglycaemia, an episode requiring the assistance of another person to actively administer carbohydrate, glucagon or take other corrective actions. a Models were adjusted for period (pre/post-switch to degludec), baseline $\mathrm{HbA}_{1 \mathrm{c}}$, gender, $\mathrm{BMI}$, duration of diabetes, age and country. Total followup time (patient-years) was 38.5 for the 4-week baseline period and 104.5 for the 12-month follow-up period. b Models were adjusted for period (pre/post-switch to

during the 12-month follow-up versus the 4-week baseline period, for both the level 1 (RR $\left.0.54[0.43 ; 0.67]_{95 \% \mathrm{CI}}\right)$ and level 2 (RR 0.56 $\left.[0.40 ; 0.79]_{95 \% \mathrm{CI}}\right)$ hypoglycaemia definitions (Fig. 1). The rate of level 3 hypoglycaemic events per PYE in patients with T2D was 0.0 in the 4-week baseline period, and 0.1 in the 12-month follow-up period; the overall number of events was insufficient to allow for statistical comparison (Fig. 1).

\section{Hypoglycaemia Rates by Patient Characteristics}

For patients with T1D, RRs demonstrated lower rates of hypoglycaemia (8-33\% lower) during the 12-month follow-up, versus the 4-week baseline period, with the majority showing a statistically significant difference (Fig. 2). However, there was no significant interaction between period and patient characteristic degludec), baseline $\mathrm{HbA}_{1 \mathrm{c}}$, gender, $\mathrm{BMI}$, duration of diabetes, bolus insulin (Yes/No), sulfonylureas or glinides (Yes/No), age and country. Total follow-up time (patientyears) was 40.8 for the 4-week baseline period and 118.8 for the 12-month follow-up period. \% percentage of patients with an event, ADA American Diabetes Association, BMI body mass index, $\mathrm{CI}$ confidence interval, $\mathrm{E}$ number of events, $R$ rate of events per patient-year of exposure, $\mathrm{N}$ number of patients with an event, T1D type 1 diabetes, T2D type 2 diabetes

group, in any of the three groups, for overall hypoglycaemia in patients with T1D (Fig. 2).

For patients with T2D, RRs demonstrated lower rates of hypoglycaemia (27-60\% lower) during the 12-month follow-up period versus the 4-week baseline period (Fig. 2). All RRs showed a statistically significant reduction in hypoglycaemia during follow-up versus baseline irrespective of baseline $\mathrm{HbA}_{1 \mathrm{c}}$ category, reason for switching and diabetes duration, except for patients with baseline $\mathrm{HbA}_{1 \mathrm{c}} \geq 8.5-<9.5 \%$ ( $\geq 69.4-<80.3 \mathrm{mmol} / \mathrm{mol}$ ) (Fig. 2). There was no significant interaction between periods and all three patient characteristic groups for overall hypoglycaemia in patients with T2D (Fig. 2).

\section{DISCUSSION}

These secondary analyses of the ReFLeCT study demonstrated that switching to degludec from other basal insulins in routine clinical practice 


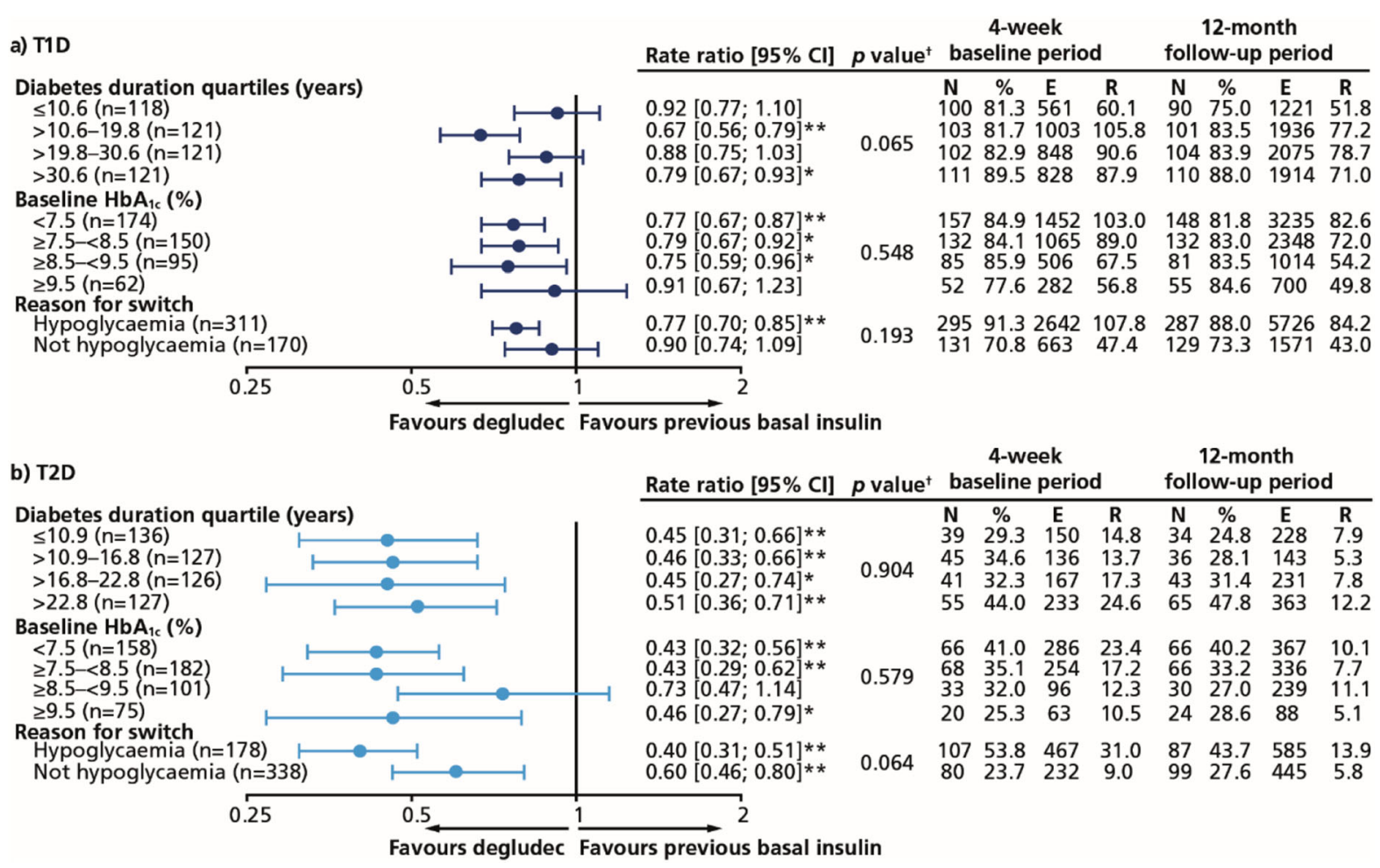

Fig. 2 Rate ratios of overall hypoglycaemia in patients with a T1D and b T2D. ${ }^{*} p<0.05 ;{ }^{* *} p<0.001 ;{ }^{\dagger} p$ values relate to a test for an interaction between period and patient characteristics for overall hypoglycaemia. Analysed using negative binomial regression models. a Models were adjusted for period (pre/post-switch to degludec) baseline $\mathrm{HbA}_{1 c}$, gender, BMI, duration of diabetes, age and country. b Models were adjusted for period (pre/postswitch to degludec), baseline $\mathrm{HbA}_{1 c}$, gender, BMI, duration of diabetes, bolus insulin (Yes/No), sulfonylureas or glinides (Yes/No), age and country. The rate ratio

was associated with lower rates of hypoglycaemia in patients with T1D or T2D, irrespective of the updated hypoglycaemia definition used, baseline glycaemic control, duration of diabetes, or reasons for switching basal insulin. These analyses corroborated the findings of the primary report that switching to degludec from other basal insulins was associated with reduced rates of overall hypoglycaemia in patients with diabetes in routine clinical practice.

RCTs have previously shown a reduced hypoglycaemia risk with degludec versus insulin glargine U100 in patients with T1D or T2D [2-5]. Patients with T1D and an $\mathrm{HbA}_{1 \mathrm{c}} \leq 10 \%$, represents the change in the 12-month follow-up period rate compared with the 4-week baseline period rate, with the significance of a test of the hypothesis that rate ratio $=1$ indicated by asterisks. Based on patient diary periods with 26-30 days. \% percentage of patients with an event, BMI body mass index, CI confidence interval, E number of events, $n$ number of patients included in fully adjusted analyses, $\mathrm{N}$ number of patients with an event, $\mathrm{R}$ rate of events per patient-year of exposure, T1D type 1 diabetes, T2D type 2 diabetes

and with $\mathrm{T} 2 \mathrm{D}$ and an $\mathrm{HbA} 1_{\mathrm{c}} \leq 9.5 \%$, were assessed, respectively, in the SWITCH-1 and -2 randomised, double-blind, treat-to-target, crossover trials; in both, patients had at least one hypoglycaemic risk factor. Significantly lower rates of overall symptomatic hypoglycaemia (BG $<3.1 \mathrm{mmol} / \mathrm{L}$ or severe) were observed with degludec versus insulin glargine U100 in both trials [3, 4]. However, as RCTs often exclude patients with certain characteristics, the analyses presented here likely reflect a broader patient cohort and demonstrate that patients at high risk of hypoglycaemia are also likely to benefit from degludec, regardless of 
long diabetes duration, low or high baseline $\mathrm{HbA}_{1 \mathrm{c}}$ levels or hypoglycaemia concerns motivating the switch to degludec. The reduced rates of hypoglycaemia after switching to degludec from other basal insulins in these groups are of clinical relevance, and suggest degludec can be used in a variety of patients with diabetes.

The use of prospective data collection was a strength of this study, reducing potential memory recall bias. Additionally, definitions for level 1, 2 and 3 hypoglycaemia were well represented in the rate of events and for the change between the baseline and follow-up periods (except for level 3 hypoglycaemia for T2D), strengthening the generalisability of these results. In this study, the rate of non-severe was higher than severe hypoglycaemia, in both the baseline and follow-up periods, reflecting the rate of events observed in the general population $[1,13,14]$. Therefore, in contrast to RCTs, these results more accurately represent patient experiences in routine clinical practice. However, as hypoglycaemia was self-reported, it cannot be excluded that patients may have underreported their hypoglycaemia, particularly severe events, owing to potential fear of losing their driving license [15]. Overall, within each patient characteristic category the results are consistent. In some patient categories, reduction in the rates of hypoglycaemia at follow-up versus baseline did not reach statistical significance, most likely owing to the small sample size or low event rate. Finally, the realworld nature of the ReFLeCT study allowed for less stringent inclusion criteria [6], therefore being more representative of clinical practice versus RCTs.

Study limitations include the observational design precluding randomisation, and that a comparator arm was not included; this makes it difficult to determine if reductions in hypoglycaemia were a treatment or study effect. As in the primary analysis, change in hypoglycaemia was not assessed by baseline insulin group. The proportion of patients receiving glargine U100 vs U300 was not recorded, and it is unclear if differences in their profiles of action may have affected results for each group when switching to degludec. There was also the potential for diary fatigue to cause incomplete reporting and bias the results. Furthermore, patients with frequent hypoglycaemia may have been overrepresented among those who agreed to participate. However, this may provide a more realistic representation of the patients likely to be switched to degludec by their physician in clinical practice.

\section{CONCLUSION}

These secondary analyses corroborated the findings of the primary ReFLeCT study, and demonstrated that, irrespective of the hypoglycaemia definition used or pre-specified patient characteristics, switching to degludec from other basal insulins was associated with reduced rates of hypoglycaemia in patients with T1D or T2D. These findings support the use of degludec in a broad range of patients with diabetes in routine clinical care.

\section{ACKNOWLEDGEMENTS}

The authors thank João Diogo Da Rocha Fernandes (Novo Nordisk A/S, Søborg, Denmark) for his review of and input to the manuscript. The authors thank the participants of the study.

Funding. This manuscript and the journal's Rapid Service Fee were funded by Novo Nordisk A/S, Søborg, Denmark.

Medical Writing and Editorial Assistance. The authors thank Alice Singleton, Chloe Harrison and Beverly La Ferla from Watermeadow Medical, an Ashfield Company (sponsored by Novo Nordisk) for providing medical writing and editorial support.

Authorship. All named authors meet the International Committee of Medical Journal Editors (ICMJE) criteria for authorship for this article, take responsibility for the integrity of the work as a whole, and have given their approval for this version to be published.

Prior Presentation. These data were previously presented at the ADA 79th Scientific 
Sessions, June 07-11 2019, San Francisco, California, USA, and at the 55th EASD Annual Meeting, September 16-20 2019, Barcelona, Spain.

Disclosures. Gian Paolo Fadini received grant support, lecture fees or advisory board fees from AstraZeneca, Boehringer Ingelheim, Eli Lilly, Novo Nordisk, Sanofi, Genzyme, Abbott, Novartis and Merck Sharp \& Dohme. Michael Feher received financial support for research, speaker meetings and consultancy from Amgen, AstraZeneca, Novo Nordisk, Eli Lilly and SanofiAventis. Michael Feher was affiliated with the University of Surrey, Guildford, UK at the time of study and is now affiliated with Nuffield Department of Primary Care Health Sciences, University of Oxford, UK. Michael Feher is still affiliated with Beta Cell Diabetes Centre, Chelsea and Westminster Hospital, London, UK. Troels Krarup Hansen and Harold W. de Valk have nothing to disclose. Esther Zimmermann and Mette Marie Koefoed are full-time employees of, and hold stock in, Novo Nordisk A/S. Ehsan Parvaresh Rizi is a full-time employee of Novo Nordisk A/S. Johan Jendle has contributed to advisory boards for AstraZeneca, Boehringer Ingelheim, Eli Lilly, Medtronic, Novo Nordisk and Sanofi, as well as chairing meetings/lectures for AstraZeneca, Boehringer Ingelheim, Eli Lilly, Nordic Infucare, Novo Nordisk and Sanofi.

Compliance with Ethics Guidelines. Informed consent was obtained from all patients, in accordance with the requirements of the Declaration of Helsinki, before any study-related activities. Independent ethics committees and institutional review boards across the participating centres reviewed and approved this study (Supplementary Table S1).

Data Availability. The datasets analysed during the current study are available from the corresponding author on reasonable request.

Open Access. This article is licensed under a Creative Commons Attribution-NonCommercial 4.0 International License, which permits any non-commercial use, sharing, adaptation, distribution and reproduction in any medium or format, as long as you give appropriate credit to the original author(s) and the source, provide a link to the Creative Commons licence, and indicate if changes were made. The images or other third party material in this article are included in the article's Creative Commons licence, unless indicated otherwise in a credit line to the material. If material is not included in the article's Creative Commons licence and your intended use is not permitted by statutory regulation or exceeds the permitted use, you will need to obtain permission directly from the copyright holder. To view a copy of this licence, visit http://creativecommons.org/licenses/by$\mathrm{nc} / 4.0 /$.

\section{REFERENCES}

1. Zekarias KL, Seaquist E. Hypoglycemia in diabetes: epidemiology, impact, prevention and treatment. Hypoglycemia-causes and occurrences: SMGroup, 2017.

2. Marso SP, McGuire DK, Zinman B, et al. Efficacy and safety of degludec versus glargine in type 2 diabetes. N Engl J Med. 2017;377:723-32.

3. Wysham C, Bhargava A, Chaykin L, et al. Effect of insulin degludec vs insulin glargine U100 on hypoglycemia in patients with type 2 diabetes: the SWITCH 2 randomized clinical trial. JAMA. 2017;318:45-56.

4. Lane W, Bailey TS, Gerety G, et al. Effect of Insulin degludec vs insulin glargine u100 on hypoglycemia in patients with type 1 diabetes: the SWITCH 1 randomized clinical trial. JAMA. 2017;318:33-44.

5. Ratner R, Gough SC, Mathieu C, et al. Hypoglycaemia risk with insulin degludec compared with insulin glargine in type 2 and type 1 diabetes: a preplanned meta-analysis of phase 3 trials. Diabetes Obes Metab. 2013;15:175-84.

6. Fadini GP, Feher M, Hansen TK, et al. Switching to degludec from other basal insulins is associated with reduced hypoglycemia rates: a prospective study. J Clin Endocrinol Metab. 2019;104:5977-90.

7. International Hypoglycaemia Study Group. Glucose concentrations of less than $3.0 \mathrm{mmol} / \mathrm{l}(54 \mathrm{mg} / \mathrm{dl})$ should be reported in clinical trials: a joint position statement of the American Diabetes Association and the European Association for the Study of Diabetes. Diabetologia. 2017;60:3-6. 
8. American Diabetes Association. 6. Glycemic targets: standards of medical care in diabetes-2019. Diabetes Care. 2019;42:S61-70.

9. Buse JB, Wolffenbuttel $\mathrm{BH}$, Herman $\mathrm{WH}$, et al. DURAbility of basal versus lispro mix 75/25 insulin efficacy (DURABLE) trial 24-week results: safety and efficacy of insulin lispro mix 75/25 versus insulin glargine added to oral antihyperglycemic drugs in patients with type 2 diabetes. Diabetes Care. 2009;32:1007-133.

10. Einhorn D, Handelsman Y, Bode BW, Endahl LA, Mersebach $\mathrm{H}$, King $\mathrm{AB}$. Patients achieving good glycemic control (HbA1c $<7 \%$ ) experience a lower rate of hypoglycemia with insulin degludec than with insulin glargine: a meta-analysis of phase $3 \mathrm{a}$ trials. Endocr Pract. 2015;21:917-26.

11. Pollom RK, Ilag LL, Lacaya LB, Morwick TM, Ortiz CR. Lilly insulin glargine versus Lantus( $\left({ }^{\circledR}\right)$ in insulin-naive and insulin-treated adults with type 2 diabetes: a randomized, controlled trial (ELEMENT 5). Diabetes Ther. 2019;10:189-203.
12. World Medical Association. World Medical Association Declaration of Helsinki: ethical principles for medical research involving human subjects. JAMA. 2013;310:2191-4.

13. Ratzki-Leewing A, Harris SB, Mequanint S, et al. Real-world crude incidence of hypoglycemia in adults with diabetes: results of the InHypo-DM study, Canada. BMJ Open Diabetes Res Care. 2018;6:e000503.

14. Sonoda N, Morimoto A, Ugi S, et al. Predictors for mild and severe hypoglycemia in insulin-treated Japanese diabetic patients. PLoS One. 2015;10: e0130584.

15. Broz J, Brabec M, Janickova Zdarska D, et al. Fear of driving license withdrawal in patients with insulintreated diabetes mellitus negatively influences their decision to report severe hypoglycemic events to physicians. Patient Prefer Adherence. 2015;9: 1367-70. 